\title{
Multiplex-heterogeneous network embedding for drug
}

\section{repositioning}

\author{
Léo Pio-Lopez ${ }^{1}$ \\ ${ }^{1}$ Aix Marseille Univ, CNRS, Centrale Marseille, I2M, Marseille, France
}

April 16, 2021

\begin{abstract}
Drug repositioning (also called drug repurposing) is a strategy for identifying new therapeutic targets for existing drugs. This approach is of great importance in pharmacology as it is a faster and cheaper way to develop new medical treatments. In this paper, we present, to our knowledge, the first application of multiplex-heterogeneous network embedding to drug repositioning. Network embedding learns the vector representations of nodes, opening the whole machine learning toolbox for a wide variety of applications including link prediction, node labelling or clustering. So far, the application of network embedding for drug repositioning focused on heterogeneous networks. Our approach for drug repositioning is based on multiplex-heterogeneous network embedding. Such method allows the richness and complexity of multiplex and heterogeneous networks to be projected in the same vector space. In other words, multiplex-heterogeneous networks aggregate different multi-omics data in the same network representation. We validate the approach on a task of link prediction and on a case study for SARS-CoV2 drug repositioning. Experimental results show that our approach is highly robust and effective for finding new drug-target associations.
\end{abstract}

Network embedding, multiplex-heterogeneous network, multi-layer network, drug repositioning, graph representation learning

\section{Introduction}

Drug repositioning (also called drug repurposing) is a strategy for identifying new therapeutic targets for existing drugs [32. This approach is of great importance in pharmacology as it is a 
faster and cheaper way to develop new medical treatments. It is particularly important for rare diseases - drug discovery is a very expensive process but rare diseases offer a very small market or in case of a pandemic, when the discovery of a fast treatment is required.

In this paper, we present to our knowledge the first application, to our knowledge, of multiplex-heterogeneous network embedding for drug repositioning. Network embedding learns the vector representations of nodes, opening the whole machine learning toolbox for a wide variety of applications including link prediction, node labelling or clustering. In addition, network embedding can handle large-scale networks, composed of millions of nodes [14. With big data, network embeddings have been applied to a wide range of networks, including for example social [23], neuronal [28] and molecular networks [29].

So far, the application of network embedding for drug repositioning focused on heterogeneous networks [25, 38, 3]. A heterogeneous network is composed of several monoplex networks with their specific type of nodes and edges and connected by bipartite interactions. In the case of drug repositioning, a heterogeneous network can be composed of drug-drug and target-target networks linked by drug-target bipartite interactions. This kind of biomedical network is usually sparse, noisy and incomplete. It appears fundamental to aggregate different sources of data to reduce the noise and add useful data to the network.

Our approach for drug repositioning is based on multiplex-heterogeneous network embedding. Such a method allows to project in the same vector space the embeddings of both drugs and targets while combining the richness and complexity of multiplex and heterogeneous networks. Indeed, multiplex networks are multi-layer networks, each layer shares the same type of nodes but their edges belong to different types. For drug repositioning, it allows to aggregate different -omics data in the same network representation. Recent methods of network embedding for drug repositioning focus on the training of a binary classifier after embedding to do link prediction and find new drug-target or drug-disease associations. In this work, we focus on biological modules by clustering the embeddings. The analysis of the cluster can also lead to the possibility of finding new drug combinations.

We validate the method on a task of link prediction and on a case study for SARS-CoV2 drug repositioning. Experimental results show that our approach is highly robust and effective to find new drug-target associations. 


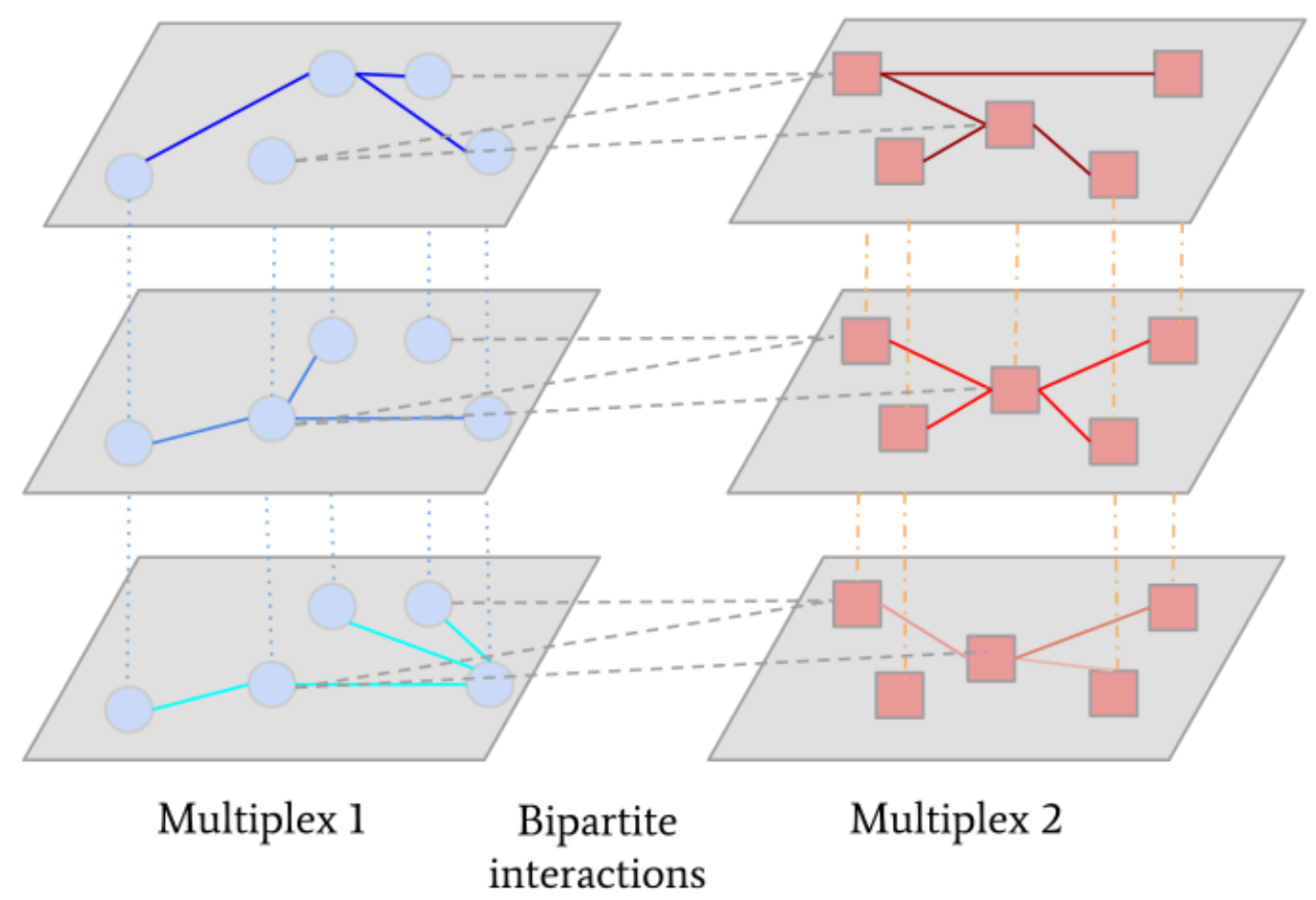

Figure 1: Representation of multiplex-heterogeneous network composed of two multiplex networks of three layers. We represented only a simplified versions of bipartite interactions (dashed lines) for a better visual understanding (bipartite interactions of one layer should link each layers of the other multiplex network). 


\section{Related work}

The application of network embedding to drug repositioning is a recent approach. So far, it focused on heterogeneous network embedding [3, 25, 38, 38, 37]. Zhou and colleagues focused on a drug-disease heterogeneous network [39. The method called NEDD, applied meta paths of different lengths to explicitly capture the similarities within drugs and diseases, by which they optimize the embeddings of drugs and diseases. NEDD uses a random forest classifier to predict novel associations between drugs and diseases.

Other approaches focused on drug-disease heterogeneous networks like in [38]. In this article, Yang et al. proposed HED to predict potential associations between drugs and diseases based on a drug-disease heterogeneous network. From the embeddings, similarly to [39], they trained an SVM binary classifier to predict new associations. Chen and colleagues introduced cross-network embedding to embed drugs, targets and diseases nodes using two heterogeneous networks, a drug-target and a drug-disease network [3].

To our knowledge, multiplex networks have never been combined with heterogeneous networks for drug repositioning. Multiplex networks are able to integrate multiple types of data and can manage network noise, which impacts the accuracy of the prediction in the case where only heterogeneous networks are used. In addition, our approach differs from the literature by focusing on biological modules to find new drug-target associations. The methods cited above used binary classification to predict new drug-target associations. Our approach focused on a cluster analysis of specific targets to find new drug-target associations.

\section{Method}

We used MultiVERSE for multiplex-heterogeneous network embedding [30. This method computes the similarities between nodes using random walks with restart on multiplex-heterogeneous network (RWR-MH) and optimizes the embeddings using Kullback-Leibler minimization. We present these two key components of the method in the next subsections.

\subsection{Random walk with restart on multiplex-heterogeneous network (RWR-MH)}

In a classical random walk (RW), an imaginary particle starts from a seed node, and explores the network, going from nodes to nodes by randomly selecting neighbour with a probability defined by its degree. In the RWR-MH algorithm, it has to travel in the two multiplex networks 
$G 1_{M}$ and $G 2_{M}$. More formally, the first multiplex network can be defined as $G 1_{M}=\left(V_{M}, E_{V_{M}}\right)$, it is a $L_{1}$-layer multiplex graph, with $n \times L_{1}$ nodes, $V_{M}=\left\{v_{i}^{\alpha}, i=1, \ldots, n, \alpha=1, \ldots, L_{1}\right\}$. The second multiplex network is a $L_{2}$-layer multiplex graph $G 2_{M}=\left(U_{M}, E_{U_{M}}\right)$ of $m \times L_{2}$ nodes with $U_{M}=\left\{u_{j}^{\alpha}, j=1, \ldots, m, \alpha_{2}=1, \ldots, L_{2}\right\}$. If there is a bipartite edge between two nodes, the particle may jump from a node in one multiplex network to the other multiplex. In multiplex-heterogeneous network, the restart can also happen in different types of node (see Figure 1) The bipartite graph is defined as $G_{B}=\left(V_{M} \cup U_{M}, E_{B}\right)$ with $E_{B} \subseteq V_{M} \times U_{M}$. With $L_{2}>L_{1}$, we can now define the multiplex-heterogeneous network $G_{M H}=\left(V_{M H}, E_{M H}\right)$ as:

$$
\begin{gathered}
V_{M H}=\left\{V_{M} \cup U_{M}\right\} \\
E_{M H}=\left\{\cup_{\alpha=1, \ldots, L_{2}} E_{B}^{[\alpha]} \cup E_{V_{M}} \cup E_{U_{M}}\right\} .
\end{gathered}
$$

The different sets of nodes $V_{M}$ and $U_{M}$ are only connected by the edges of the bipartite graph. It is to note that the bipartite edges should link nodes with every layer of the multiplex graphs.

Therefore, we can now define the evolution of the probability distribution of random walk with restart [24]: $\mathbf{p}_{t}=\left(p_{t}(v)\right)_{v \in V_{M H}}$. This distribution can be described as follows:

$$
\mathbf{p}_{t+1}^{T}=(1-r) M \mathbf{p}_{t}^{T}+r \mathbf{p}_{0}^{T}
$$

where $M$ denotes a transition matrix that is the column normalization of $A_{M H}$, which is the multiplex-heterogeneous transition matrix. The vector $\mathbf{p}_{0}$ is the initial probability distribution. With a probability $r \in(0,1)$, the particle can jump back to the initial node(s), known as seed(s), at each step. The stationary distribution of Equation (1) represents the probability for the particle to be located at a specific node for an infinite amount of time [24].

Finally, this distribution can be interpreted as a similarity between the seed(s) and the other nodes. We use it to optimize the embeddings.

\subsection{Learning objective}

In the context of MultiVERSE, we need to compute a similarity in the multiplex-heterogeneous network $G_{M H}$ denoted $\operatorname{sim}_{G}: V_{M H} \times V_{M H} \rightarrow \mathbb{R}$ and defined as follows:

$$
\forall v \in V_{M H}, \sum_{u \in V} \operatorname{sim}_{G}(v, u)=1
$$


Therefore, $\operatorname{sim}_{G}(v,$.$) , the similarity for any node v$ of the multiplex-heterogeneous network is expressed as a probability distribution. As $\operatorname{sim}_{G}(v,$.$) is defined as a probability distribution,$ MultiVERSE applies a softmax function to obtain the normalized similarity distribution in the embedding or vector space. Formally, $w_{i}$ is defined as the embeddings of node $i$ in the embedding space, the similarity between two nodes embeddings $w_{u}$ and $w_{v}$ is defined as the dot product $w_{u} \cdot w_{v}^{T}$ and:

$$
\operatorname{sim}_{E m b}(v, .)=\frac{\exp \left(w_{v} \cdot w^{T}\right)}{\sum_{i=1}^{n} \exp \left(w_{v} \cdot w_{i}\right)} .
$$

The aim of MultiVERSE is to approximate the similarity distribution in the embedding space, denoted $\operatorname{sim}_{E m b}: V_{M H} \times V_{M H} \rightarrow \mathbb{R}$ such as $\forall v \in V_{M H}, \operatorname{sim}_{G}(v,.) \approx \operatorname{sim}_{E m b}(v$.$) . This learning$ phase is performed using Kullback-Leibler minimization between the two similarities:

$$
\sum_{v \in V_{M}} K L\left(\operatorname{sim}_{G}(v, .) \| \operatorname{sim}_{E m b}(v, .)\right)
$$

By keeping only the terms related to $\operatorname{sim}_{E m b}$ as $\operatorname{sim}_{G}$ is constant, we obtain the following objective function:

$$
\mathcal{L}=-\sum_{v \in V_{M}} \operatorname{sim}_{G}(v, .) \log \left(\operatorname{sim}_{E m b}(v, .)\right)
$$

At each iteration, as $\operatorname{sim}_{E m b}$ is defined as a softmax function, it is necessary to normalize it over all the nodes of the network, which is computationally heavy. As in the original MultiVERSE algorithm, we used Noise Contrastive Estimation (NCE) to approximate the computations 12 .

To sum up, in this framework, the similarity in the multiplex-heterogenous network is computed using Random Walks with Restart on Multiplex-Heterogeneous (RWR-MH) networks [36. And MultiVERSE applies Kullback-Leibler minimization to optimize the embeddings. The RWR-MH parameters are the same values used in the original study $\left(r=0.7, \boldsymbol{\tau}_{\mathbf{1}}=\left(1 / L_{1}, 1 / L_{1}, \ldots, 1 / L_{1}\right)\right.$, $\left.\boldsymbol{\tau}_{\mathbf{2}}=\left(1 / L_{2}, 1 / L_{2}, \ldots, 1 / L_{2}\right), \delta=0.5, \lambda=0.5, \eta=0.5\right)[36]$.

The reader can refer to the original article for more details on the method and particular implementation of the algorithm [30] 


\section{Experimental setup}

\subsection{Datasets}

We used different datasets to construct the multiplex-heterogeneous network. In this work, it is composed of one human molecular multiplex network (3 layers), one drug multiplex network (4 layers) and a heterogeneous drug-target network (corresponding to the bipartite interactions):

- Human molecular multiplex network: This network is a molecular network, extracted from [30] with additional SARS-CoV2 interactions, consisting of 3 layers: 1) A proteinprotein interaction (PPI) layer (14704 nodes, 122211 edges) which is the integration of 4 datasets: APID (apid.dep.usal.es) (Level 2, human only), Hi-Union, Lit-BM (http: //www.interactome-atlas.org/download) and the additional interactions of SARS-CoV2 from [11] in the PPI layer. 2) The second layer (7926 nodes, 194500 edges) is a pathways layer corresponding to the human Reactome data [5] extracted from NDEx [31]. 3) The last layer (8537 nodes, 63561 edges) is a molecular complexes layer constructed from the fusion of Hu.map [8] and Corum [10].

- Drug multiplex network: We constructed the following 4-layers drug multiplex network: (1) the first layer (877 nodes, 2795 edges) has been extracted from Bionetdata (https://rdrr io/cran/bionetdata/man/DD.chem.data.html) and the edges correspond to Tanimoto chemical similarities between drugs if superior to $0.6,(2)$ the second layer (362 nodes, 678 edges) comes from [4] and the edges are based on drug combinations as reported in clinical data, (3) the third layer (658 nodes, 13397 edges ) is the adverse drug-drug interactions network available in [4]. (4) The last layer (4298 nodes, 48593 edges) is the projected drugtarget network on the drug layer from Biosnap (http://snap.stanford.edu/biodata/ datasets/10002/10002-ChG-Miner.html). In this layer, if the Jaccard Index between the neighborhoods of two drug nodes in the original drug-target network is superior to 0.4 , an edge between two drugs is created in the projected network. Therefore, two drugs are linked if they share a similar set of genes.

- Bipartite network: We extracted the drug-target bipartite network from [4]. It has 15030 bipartite interactions between 4412 drugs and 2255 protein targets. 


\subsection{Evaluation of the approach}

\subsubsection{Link prediction}

Similarly to [30], we used link prediction to evaluate the quality of the embeddings and validate our approach for drug repositioning. The link prediction pipeline is the following: first, we remove randomly $30 \%$ of the bipartite edges to obtain a training network, we then train a Random Forest on this training network, and test on the $30 \%$ removed edges. In order to train the binary classifier, we have to apply operators (Hadamard, Weighted-L1, Weighted-L2, Average and cosine) to the embeddings.

The aim of this validation task is to assess the quality of the embeddings in order to find drug-target associations. Direct comparisons with other methods are not possible as, to our knowledge, there is no other multiplex-heterogeneous network embedding method projecting both types of nodes in the literature.

\subsubsection{Case study on SARS-CoV2 drug repositioning}

The second approach we used for validation is to test the method on a case study, here the SARS-CoV2 drug repositioning. Indeed, in these times of pandemic, there is abundant literature on the efficacy of different drugs, both in vitro and in vivo. In addition, with the appearance of variants resistant to vaccines, drug repositioning is still particularly relevant.

In order to find new drug-target associations, we focused on biological modules. Once MultiVERSE has been applied to the drug-target multiplex-heterogeneous network, we used a clustering method on the embeddings and analysed the clusters. This approach allows us to find new drug-target associations but also possible drug combinations as they are included in the same drug-target modules. The clustering method we apply is spherical k-means [2] with $k=500$ applied on the embedding. We analyse the 27 clusters corresponding to the SARS-CoV2 proteins [11]. We then confront our results to the clinical and biological literature to evaluate the usefulness and quality of our predictions.

\section{$5 \quad$ Experimental results}

\subsection{Results on link prediction}

MultiVERSE has a score of ROC-AUC superior to 0.9 with the Hadamard, Average and Cosine operators (see Table 1), meaning that the method can predict with high precision the removed 


\begin{tabular}{cc}
\hline Operators & ROC-AUC \\
\hline Hadamard & $\mathbf{0 . 9 7 8} \pm \mathbf{0 . 0 0 0 6}$ \\
Weighted-L1 & $0.708 \pm 0.011$ \\
Weighted-L2 & $0.709 \pm 0.01$ \\
Average & $\mathbf{0 . 9 6 2} \pm \mathbf{0 . 0 0 4}$ \\
Cosine & $\mathbf{0 . 9 3 2} \pm \mathbf{0 . 0 0 7}$
\end{tabular}

Table 1: ROC-AUC scores for link prediction using MultiVERSE. Link predictions are computed for the bipartite interactions of the multiplex-heterogeneous networks. The scores higher than 0.9 are highlighted in bold.

$30 \%$ of drug-target links from the corresponding multiplex-heterogeneous networks.

The variance is very small for all operators. The network embedding method is highly robust and steady across each run of the link prediction evaluation test.

\subsection{Cluster analysis of SARS-CoV2 proteins}

In the clusters of the different SARS-CoV2 proteins, we found 88 molecules, out of which 33 are already FDA-approved. Given length constraints, we will present here the most interesting of them.

We found two drugs of interest in the nsp1 protein cluster: cladribine and gallium maltolate. Cladribine is used for multiple sclerosis and has been associated with mild or no symptoms after COVID-19 infection [6. Gallium maltolate has also in vitro activity against the virus [1].

In the cluster of the nsp6 protein, we have the anti-malarial drug mefloquine that has in vitro inhibition of SARS-CoV2 9]. Other anti-malarial drugs are present in this cluster like Halofantrine, that have been proposed for COVID-19 repositioning [33] or Voacamine. Amodiaquine is also a drug that could be a target for this disease [13. We also found dronedarone that has also been identified as an active inhibitor of the virus [20].

We also found glutathione in the nsp5 C145A protein cluster. This molecule could address the cytokine storm syndrome [16].

In the $\mathrm{N}$ protein cluster, we have a repositioning target with S-oxy-L-cysteine. It is a member of the family of L-alpha-amino acids. L-cysteine in combination with vitamin D has been shown to reduce mortality associated with COVID-19 in African Americans [19. We also found in this cluster a preclinical molecule, the sanglifehrin A, which glue linking IMPDH with cyclophilin A, which itself is involved in viral capsid packaging.

We obtained one interesting drug in the cluster including the orf3b protein of COVID-19. We found the FDA-approved drug ezetimibe a a potential target for repositioning. It has been 
reported that patients taking this molecule have significantly reduced odds for SARS-CoV-2 hospitalization [18].

In the orf3a protein cluster, we identified the lumichrome molecule, a constituent of honey that would be an inhibitor of the virus [15]. We have also the riboflavin drug which has been shown to inactivate the virus [22].

Several potential targets for repositioning are in the nsp14 protein cluster of COVID-19. We have the AICA ribonucleotide, an AMP-activated protein kinase activator it has been shown to be effective as a treatment against influenza [27]. In addition, another AMPK activator, metformin, has been associated with decreased mortality in COVID-19 [26]. In this cluster, we also have mycophenolate mofetil which is transformed in its active form the mycophenolic acid as a target for repositioning. This acid is known to inhibit dengue virus and it has been shown that it is active against COVID-19 [21] and has been proposed as a treatment in combination with interferon [7. We also have the ribarivin anti-viral drug that has been used clinically for COVID-19 treatments in combination with other drugs [17.

The valproic acid is in the cluster of the nsp5 protein of COVID-19. This compound has been proposed a a potential treatment as it reduces ACE2 expression in endothelial cells [34. We also indentified panobinostat, a histone deacetylase inhibitor that could suppress ACE2 and ABO of COVID-19 [35].

\section{Conclusion and perspectives}

We presented the first application of multiplex-heterogeneous network embedding for drug repositioning. The method combines for the first time the richness of multiplex networks with the complexity of heterogeneous networks in order to find new drug targets. We tested the quality of the embeddings on link prediction and showed the method is highly robust to find drug-target associations for COVID-19. We also found several targets that have have anti-viral properties against SARS-CoV2 in vitro or in vivo.

We also identified several other targets for repositioning with this approach. We have for example different anti-cancer drugs like vorinostat or pracinostat in the nsp5 cluster for example that needs further investigations. We also have all the other molecules that are not FDA-approved we included in the multiplex-heterogeneous network.

There are several perspectives to this work. In this article, we projected drugs and targets in the same vector space. An interesting extension would be to project drugs, targets and diseases 
in the same vector space using either cross-network embedding [3] for multiplex-heterogeneous networks or by extending RWR to 3 multiplex (molecular, drug and disease) and 2 bipartite networks (drug-target and drug-disease for example).

\section{Acknowledgements}

We thank Alberto Valdeolivas and Anaïs Baudot for discussions on a early phase of this work. We are grateful to Maxime Lucas for helpful comments on a first draft of this work.

\section{References}

[1] Bernstein, L. R., And Zhang, L. Gallium maltolate has in vitro antiviral activity against sars-cov-2 and is a potential treatment for covid-19. Antiviral Chemistry and Chemotherapy 28 (2020), 2040206620983780.

[2] Buchta, C., Kober, M., Feinerer, I., And Hornik, K. Spherical k-means clustering. Journal of Statistical Software 50, 10 (2012), 1-22.

[3] Chen, H., Cheng, F., And Li, J. idrug: Integration of drug repositioning and drug-target prediction via cross-network embedding. PLoS computational biology 16, 7 (2020), e1008040.

[4] Cheng, F., Kovács, I. A., and Barabási, A.-L. Network-based prediction of drug combinations. Nature communications 10, 1 (2019), 1-11.

[5] Croft, D., Mundo, A. F., Haw, R., Milacic, M., Weiser, J., Wu, G., Caudy, M., Garapati, P., Gillespie, M., Kamdar, M. R., et Al. The reactome pathway knowledgebase. Nucleic acids research 42, D1 (2014), D472-D477.

[6] De Angelis, M., Petracca, M., Lanzillo, R., Morra, V. B., and Moccia, M. Mild or no covid-19 symptoms in cladribine-treated multiple sclerosis: Two cases and implications for clinical practice. Multiple sclerosis and related disorders 45 (2020), 102452.

[7] Dowran, R., Nabavi, S. F., Habtemariam, S., Banach, M., Shahmohamadnejad, S., Cismaru, C. A., Berindan-Neagoe, I., Sahebnasagh, A., and Nabavi, S. M. Various interferon (ifn)-inducible transmembrane (ifitm) proteins for covid-19, is there a role for the combination of mycophenolic acid and interferon? Biochimie 177 (2020), 50-52. 
[8] Drew, K., Lee, C., Huizar, R. L., Tu, F., Borgeson, B., McWhite, C. D., Ma, Y., WAllingford, J. B., AND Marcotte, E. M. Integration of over 9,000 mass spectrometry experiments builds a global map of human protein complexes. Molecular systems biology 13, $6(2017)$.

[9] Gendrot, M., Duflot, I., Boxberger, M., Delandre, O., Jardot, P., Le Bideau, M., Andreani, J., Fonta, I., Mosnier, J., Rolland, C., Et Al. Antimalarial artemisininbased combination therapies (act) and covid-19 in africa: In vitro inhibition of sars-cov-2 replication by mefloquine-artesunate. International journal of infectious diseases 99 (2020), $437-440$.

[10] Giurgiu, M., Reinhard, J., Brauner, B., Dunger-Kaltenbach, I., Fobo, G., Frishman, G., Montrone, C., And Ruepp, A. Corum: the comprehensive resource of mammalian protein complexes-2019. Nucleic acids research 47, D1 (2019), D559-D563.

[11] Gordon, D. E., Jang, G. M., Bouhaddou, M., Xu, J., Obernier, K., White, K. M., O’Meara, M. J., Rezelu, V. V., Guo, J. Z., Swaney, D. L., et Al. A sars-cov-2 protein interaction map reveals targets for drug repurposing. Nature (2020), 1-13.

[12] Gutmann, M., And Hyvärinen, A. Noise-contrastive estimation: A new estimation principle for unnormalized statistical models. In Proceedings of the Thirteenth International Conference on Artificial Intelligence and Statistics (2010), pp. 297-304.

[13] Hagar, M., Ahmed, H. A., Aljohani, G., And Alhaddad, O. A. Investigation of some antiviral n-heterocycles as covid 19 drug: Molecular docking and dft calculations. International Journal of Molecular Sciences 21, 11 (2020), 3922.

[14] Hamilton, W. L., Ying, R., And Leskovec, J. Representation learning on graphs: Methods and applications. IEEE Data Engineering Bulletin (2017).

[15] Hashem, H. E. In silico approach of some selected honey constituents as sars-cov-2 main protease (covid-19) inhibitors. Eurasian J. Med. Oncol 4 (2020), 196-200.

[16] Horowitz, R. I., Freeman, P. R., And Bruzzese, J. Efficacy of glutathione therapy in relieving dyspnea associated with covid-19 pneumonia: A report of 2 cases. Respiratory medicine case reports 30 (2020), 101063.

[17] Hung, I. F.-N., Lung, K.-C., Tso, E. Y.-K., Liu, R., Chung, T. W.-H., Chu, M.-Y., Ng, Y.-Y., Lo, J., Chan, J., Tam, A. R., ET AL. Triple combination of interferon beta-1b, 
lopinavir-ritonavir, and ribavirin in the treatment of patients admitted to hospital with covid-19: an open-label, randomised, phase 2 trial. The Lancet 395, 10238 (2020), 1695-1704.

[18] Israel, A., Schäffer, A. A., Cicurel, A., Feldhamer, I., Tal, A., Cheng, K., Sinha, S., Schiff, E., Lavie, G., And Ruppin, E. Large population study identifies drugs associated with reduced covid-19 severity. medRxiv (2020).

[19] Jain, S. K., and Parsanathan, R. Can vitamin d and l-cysteine co-supplementation reduce 25 (oh)-vitamin d deficiency and the mortality associated with covid-19 in african americans? Journal of the American College of Nutrition 39, 8 (2020), 694-699.

[20] Jeon, S., Ko, M., Lee, J., Choi, I., Byun, S. Y., Park, S., Shum, D., And Kim, S. Identification of antiviral drug candidates against sars-cov-2 from fda-approved drugs. Antimicrobial agents and chemotherapy 64, 7 (2020).

[21] Kato, F., Matsuyama, S., Kawase, M., Hishiki, T., Katoh, H., and Takeda, M. Antiviral activities of mycophenolic acid and imd-0354 against sars-cov-2. Microbiology and Immunology 64, 9 (2020), 635-639.

[22] Keil, S. D., Ragan, I., Yonemura, S., Hartson, L., Dart, N. K., and Bowen, R. Inactivation of severe acute respiratory syndrome coronavirus 2 in plasma and platelet products using a riboflavin and ultraviolet light-based photochemical treatment. Vox sanguinis 115,6 (2020), 495-501.

[23] Liao, L., He, X., Zhang, H., and Chua, T.-S. Attributed social network embedding. IEEE Transactions on Knowledge and Data Engineering 30, 12 (2018), 2257-2270.

[24] Lovász, L. Random walks on graphs: A survey. Combinatorics Paul Erdos is Eighty 2, Volume 2 (1993), 1-46.

[25] Lu, Z., Wang, Y., Zeng, M., And Li, M. Hnedti: Prediction of drug-target interaction based on heterogeneous network embedding. In 2019 IEEE International Conference on Bioinformatics and Biomedicine (BIBM) (2019), IEEE, pp. 211-214.

[26] Luo, P., Qiu, L., Liu, Y., Liu, X.-L., Zheng, J.-L., Xue, H.-Y., Liu, W.-H., Liu, D., AND LI, J. Metformin treatment was associated with decreased mortality in covid-19 patients with diabetes in a retrospective analysis. The American journal of tropical medicine and hygiene 103, 1 (2020), 69-72. 
[27] Luzi, L., AND Radaelli, M. G. Influenza and obesity: its odd relationship and the lessons for covid-19 pandemic. Acta diabetologica 57, 6 (2020), 759-764.

[28] Ma, G., Lu, C.-T., He, L., Philip, S. Y., And Ragin, A. B. Multi-view graph embedding with hub detection for brain network analysis. In 2017 IEEE International Conference on Data Mining (ICDM) (2017), IEEE, pp. 967-972.

[29] Nelson, W., Zitnik, M., Wang, B., Leskovec, J., Goldenberg, A., And Sharan, R. To embed or not: network embedding as a paradigm in computational biology. Frontiers in genetics 10 (2019).

[30] Pio-Lopez, L., Valdeolivas, A., Tichit, L., Remy, É., And Baudot, A. Multiverse: a multiplex and multiplex-heterogeneous network embedding approach. arXiv preprint arXiv:2008.10085 (2020).

[31] Pratt, D., Chen, J., Welker, D., Rivas, R., Pillich, R., Rynkov, V., Ono, K., Miello, C., Hicks, L., Szalma, S., ex Al. Ndex, the network data exchange. Cell systems 1, 4 (2015), 302-305.

[32] Pushpakom, S., Iorio, F., Eyers, P. A., Escott, K. J., Hopper, S., Wells, A., Doig, A., Guilliams, T., Latimer, J., McNamee, C., et Al. Drug repurposing: progress, challenges and recommendations. Nature reviews Drug discovery 18, 1 (2019), $41-58$.

[33] Schlagenhauf, P., Grobusch, M. P., Maier, J. D., and Gautret, P. Repurposing antimalarials and other drugs for covid-19. Travel medicine and infectious disease 34 (2020), 101658.

[34] Singh, S., AND Singh, K. Valproic acid in prevention and treatment of covid-19. Authorea Preprints (2020).

[35] Takahashi, Y., Hayakawa, A., Sano, R., Fukuda, H., Harada, M., Kubo, R., Okawa, T., And Kominato, Y. Histone deacetylase inhibitors suppress ace2 and abo simultaneously, suggesting a preventive potential against covid-19. Scientific Reports 11, 1 (2021), 1-9.

[36] Valdeolivas, A., Tichit, L., Navarro, C., Perrin, S., Odelin, G., Levy, N., Cau, P., Remy, E., And Baudot, A. Random walk with restart on multiplex and heterogeneous biological networks. Bioinformatics 35, 3 (2018), 497-505. 
[37] Xiong, Y., Guo, M., Ruan, L., Kong, X., Tang, C., Zhu, Y., and Wang, W. Heterogeneous network embedding enabling accurate disease association predictions. $B M C$ medical genomics 12, 10 (2019), 1-17.

[38] Yang, K., Zhao, X., Waxman, D., and Zhao, X.-M. Predicting drug-disease associations with heterogeneous network embedding. Chaos: An Interdisciplinary Journal of Nonlinear Science 29, 12 (2019), 123109.

[39] Zhou, R., Lu, Z., Luo, H., Xiang, J., Zeng, M., And Li, M. Nedd: a network embedding based method for predicting drug-disease associations. BMC bioinformatics 21, 13 (2020), 1-12. 